\title{
Caracterización de lesiones asociadas a microcalcificacio- nes BI-RADS 4A, en 11 años de biopsias estereotáxicas
} \author{
Jocelyn Gálvez $T^{(1)}$. \\ 1. Departamento de Imagenología Mamaria, Clínica Alemana de Santiago. \\ Facultad de Medicina Clínica Alemana - Universidad del Desarrollo. Santiago, Chile. \\ 2. Servicio de Imagenología, Hospital Guillermo Grant Benavente, Concepción. Chile.
}

Drs. Marcela Uchida $S^{(1)}$, Monserrat Fernández $\mathbf{G}^{(2)}$, Miguel A. Pinochet $T^{(1)}$, TMs. María Paz Durán M(1),

\section{Characterization of lesions associated with microcalcifications BI-RADS 4A over a 11-year period of stereotactic breast biopsies}

\begin{abstract}
Substantial advances in breast imaging techniques, especially developments in digital mammography, have led to early detection of breast cancer. It is well-known that microcalcifications are present in approximately 55\% of nonpalpable breast malignancies and are responsible for the detection of $85-90 \%$ of cases of ductal carcinoma in situ (DCIS) through mammographic screening.

We evaluated the types of associated lesions and the percentage of malignancy in BI-RADS 4A subcategory (low suspicion of malignancy), by performing a database review of stereotactic biopsies of microcalcifications categorized as BI-RADS 4A, between September 1999 and January 2011, which accounted for 21.4\% of biopsied microcalcifications in a total of 159 women. Histological findings corresponded to benign lesions in $43.5 \%$, high-risk lesions in $46.5 \%$, and malignant tumors in 10\%. Concerning the latter (16 biopsies), $81.3 \%$ were DCIS and $18.7 \%$ corresponded to infiltrating ductal carcinoma (IDC). The PPV of BI-RADS 4 A category was $13 \%$, a value consistent with that described in the literature.

Microcalcifications BI-RADS 4A exhibit low suspicion of malignancy, since they mostly correspond to benign lesions (90\%). Subcategory $4 A$ constitutes an important ancillary diagnostic tool for a more accurate assessment of lesions suspicious for malignancy; therefore, we strongly recommend its use.

Keywords: BI-RADS 4A, Microcalcifications, Stereotactic biopsy.
\end{abstract}

Resumen: El continuo avance en las técnicas de imágenes mamarias, especialmente el desarrollo de la mamografía digital, ha permitido detectar cáncer mamario en etapa precoz. Se sabe que las microcalcificaciones están presentes en el 55\% de los cánceres no palpables y corresponden al $85-90 \%$ de los carcinomas ductales in situ (CDIS) que se detectan con mamografía de screening.

Hemos evaluado el tipo de lesiones asociadas y el porcentaje de malignidad de la subcategoría BI-RADS $4 A$ (baja sospecha de malignidad), realizando una revisión de la base de datos de las biopsias estereotáxicas por microcalcificaciones categorizadas BI-RADS 4A entre septiembre 1999 y enero 2011 y que alcanzaron al 21,4\% del total de las microcalcificaciones biopsiadas, en un total de 159 mujeres. Los resultados histológicos correspondieron a lesiones benignas en el $43,5 \%$, lesiones de alto riesgo en el 46,5\% y malignas en $10 \%$. De las lesiones malignas (16 biopsias), el $81,3 \%$ fue CDIS y el 18,7\% carcinoma ductal infiltrante (CDI). EI VPP de la categoría BI-RADS 4 A fue de 13\%, concordante con la literatura.

Las microcalcificaciones BI-RADS $4 A$ son de baja sospecha de malignidad, correspondiendo en su gran mayoría (90\%) a lesiones benignas. La subdivisión en 4 A representa una herramienta que facilita un mejor manejo clínico de las pacientes, por lo que recomendamos su utilización.

Palabras clave: Biopsia estereotáxica, BI-RADS 4 A, Microcalcificaciones.

Uchida M y cols. Caracterización de lesiones asociadas a microcalcificaciones BI-RADS 4A, en 11 años de biopsias estereotáxicas Rev Radiol 2012; 18(1): 30-35.

Correspondencia: Dra. Monserrat Fernández / monsefer@vtr.net

Trabajo recibido el 25 de diciembre de 2011, aceptado para publicación el 19 de marzo de 2012.

\section{Introducción}

Ha habido un importante avance en las técnicas de imágenes mamarias, especialmente con el desarrollo de la mamografía full digital, lo que permite pesquisa de lesiones en etapa subclínica y por ende un diagnóstico precoz de cáncer de mama. Se sabe que las microcalcificaciones están presentes en el $55 \%$ de los cánceres no palpables ${ }^{(1)}$ y corresponden 
al $85-90 \%$ de los carcinomas ductales in situ (CDIS) que se detectan con mamografía de screening ${ }^{(2)}$.

Se generó, entonces, la necesidad de clasificar y caracterizar las lesiones, entre ellas las microcalcificaciones, con el fin de predecir su potencial riesgo de malignidad. Surgió así en el año 1992 el BI-RADS (Breast Imaging Reporting and Data System), realizado por el Colegio Americano de Radiología(3), que comprende las categorías del 0 al 6 . Se recomienda biopsia en aquellas lesiones BI-RADS 4 y 5 , cuya probabilidad de malignidad es de $23-30 \%$ y $95 \%$ respectivamente ${ }^{(4,5)}$.

Desde el año 2003 en su cuarta edición, BI-RADS divide a la categoría BI-RADS 4 en tres subgrupos 4A (baja sospecha de malignidad), 4B (sospecha intermedia de malignidad) y $4 \mathrm{C}$ (moderada sospecha, pero no clásico para malignidad), lo que ha permitido según algunos estudios aumentar el valor predictivo positivo (VPP) de la categoría BI-RADS $4^{(6,7)}$ y estratificar el riesgo de malignidad en el amplio espectro de microcalcificaciones BI-RADS $4^{(8)}$.

\section{Objetivo}

Evaluar el tipo de lesiones asociadas y el porcentaje de malignidad de la subcategoría BI-RADS $4 A$ en biopsias estereotáxicas (BE) por microcalcificaciones.

\section{Material y método}

Se realizó una revisión retrospectiva, utilizando base de datos Filemaker Pro 8.5, seleccionando aquellas $B E$ por microcalcificaciones categorizadas BI-RADS 4A. La búsqueda fue hecha entre septiembre de 1999 y enero de 2011.

Las imágenes fueron analizadas en placas hasta marzo del año 2007 y luego en dos estaciones de trabajo (MA 3000 AGFA) con monitores de 5 megapíxeles, conectadas al sistema RIS-PACS. Todas las pacientes contaban con las proyecciones, medio oblicua lateral y cráneo-caudal (CC) y magnificaciones en cráneo-caudal y lateral estricta (LT). La lectura de las mamografías y las biopsias fueron realizadas por siete radiólogos especialistas en imágenes mamarias con más de cinco años de experiencia.

Se utilizó mesa de estereotaxia (Lorad Hologic Com., model Multicare Platinium Asy-00072), con sistema mammotome, (Control Module with SmartVac, Ethicon Endo-Surgery.Inc.J\&J Com.) con aguja 8G y Suros (Hologic ATEC Breast biopsy and excision system, Model Saphire TM Unit, Handpiece), con aguja 9G.

Se dejó clip metálico de marcación en lugar de extracción de las microcalcificaciones y se efectuó control mamográfico post- procedimiento.

El análisis histológico se llevó a cabo por un mismo patólogo de la institución.

Se evaluaron las histologías de la BE y de la biopsia quirúrgica en los casos en que la paciente fue operada.
Las lesiones se dividieron en benignas, malignas y lesiones de alto riesgo, incluyéndose en esta última categoría a la hiperplasia ductal atípica (HDA), atipia epitelial plana (AEP), neoplasia lobulillar (LIN) grado I, II y III y lesión mucocele like.

Todas las pacientes firmaron consentimiento informado antes de realizarse la BE.

Se evaluaron datos demográficos, características de las lesiones, del procedimiento y resultado histológico.

\section{Resultados}

En el periodo analizado se realizaron $751 \mathrm{BE}$ por microcalcificaciones.

Las microcalcificaciones biopsiadas fueron clasificadas como BI-RADS 2(1), BI-RADS 3 (15), BIRADS 4 (493), BI-RADS 4A (161), BI-RADS 4B (52), BI-RADS 4C (17) y como BI-RADS 5 (12) (Tabla I).

Tabla I. Clasificación según BI-RADS de microcalcificaciones en BE

\begin{tabular}{|lcc|} 
BI-RADS & No biopsias & $\%$ \\
\hline 1 & 0 & 0 \\
2 & 1 & 0,1 \\
3 & 15 & 2,0 \\
4 & 493 & 65,6 \\
$4 \mathrm{~A}$ & 161 & 21,4 \\
4B & 52 & 6,9 \\
4C & 17 & 2,3 \\
5 & 12 & 1,7 \\
Total & $\mathbf{7 5 1}$ & $\mathbf{1 0 0}$ \\
\hline
\end{tabular}

Las microcalcificaciones BI-RADS 4A (161) correspondieron al $21,4 \%$ del total de las microcalcificaciones biopsiadas, en un total de 159 mujeres.

La edad promedio de las pacientes fue 51,9 años con un rango entre 34 a 76 años. El tamaño promedio de los focos de microcalcificaciones biopsiados fue $9,3 \mathrm{~mm}$ con un rango entre 2 a $50 \mathrm{~mm}$.

En cada biopsia se extrajeron entre 6 a 40 especímenes, con un promedio de 16,7. En todos los casos se realizó mamografía digital de los especímenes. (Figura 1 a y 1 b) y se logró extracción completa en $52 \%$ de los casos (Figura 2).

Hubo complicaciones menores en tres pacientes $(1,9 \%)$, que correspondieron a sangrado en la zona de punción, el cual fue controlado al finalizar el procedimiento, sin repercusiones posteriores.

Los resultados histológicos (Gráfico 1) correspondieron a lesiones benignas en 70 casos $(43,5 \%)$ (Figura $3 a$ y $3 b$ ), lesiones de alto riesgo en 75 casos $(46,5 \%)$ (Figura $4 a$ y $4 b)$ y malignas en 16 biopsias (10\%) (Figura 5). 


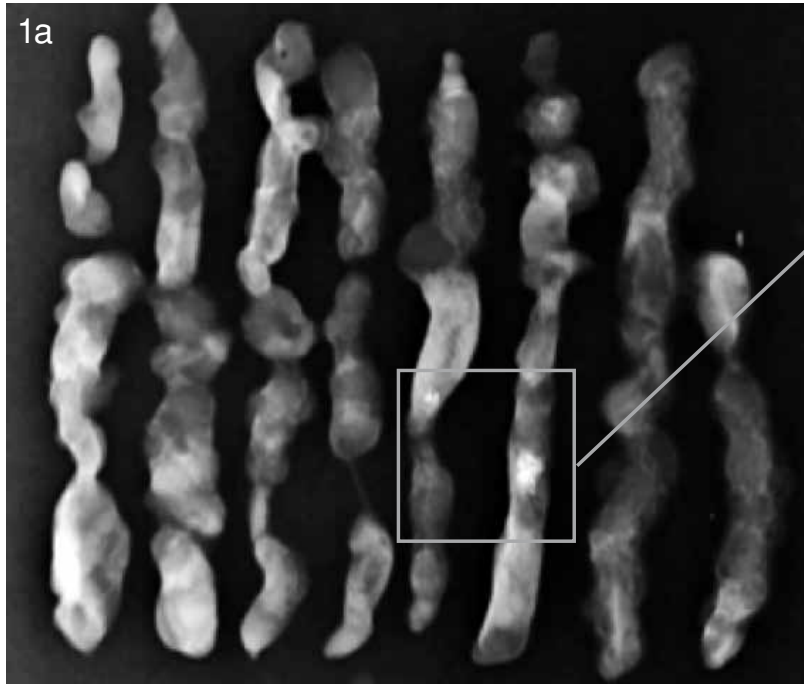

Figura 1a. Mamografía digital de los especímenes extraídos en $B E$, que muestra microcalcificaciones.

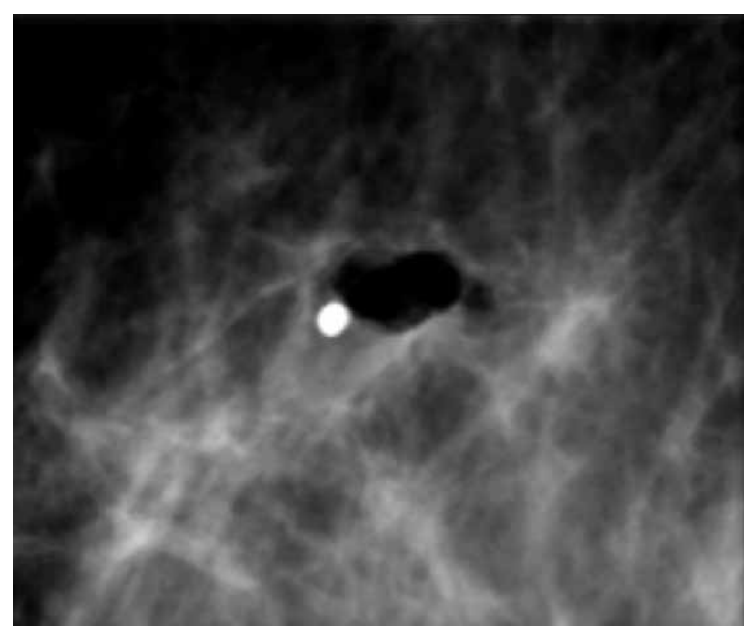

Figura 2. Spot digital, donde no se aprecian microcalcificaciones residuales y se reconoce clip metálico en el sitio de biopsia.

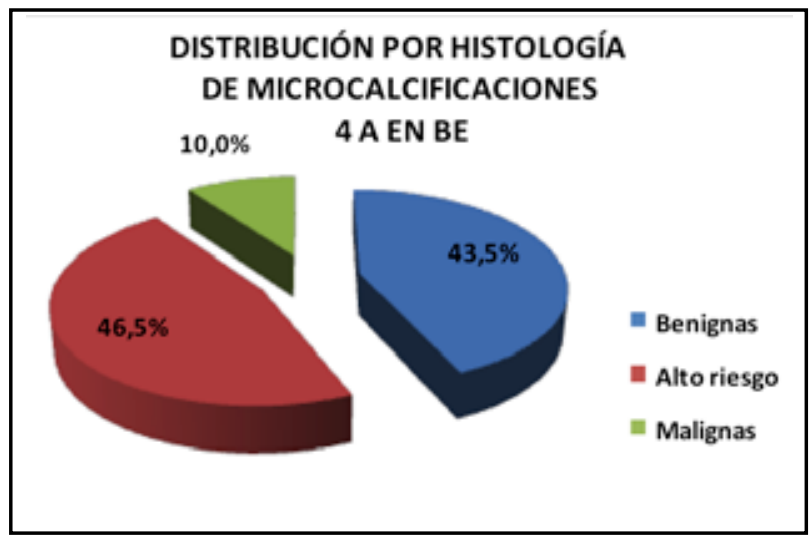

Gráfico 1.



Figura 1b. Acercamiento de microcalcificaciones en especímenes de BE.


Figura $3 \boldsymbol{a}$ y $\mathbf{3 b}$. Magnificaciones en proyecciones CC y $L T$ de grupo de microcalcificaciones catalogadas como BIRADS $4 A$, cuya histología en la BE fue benigna. 

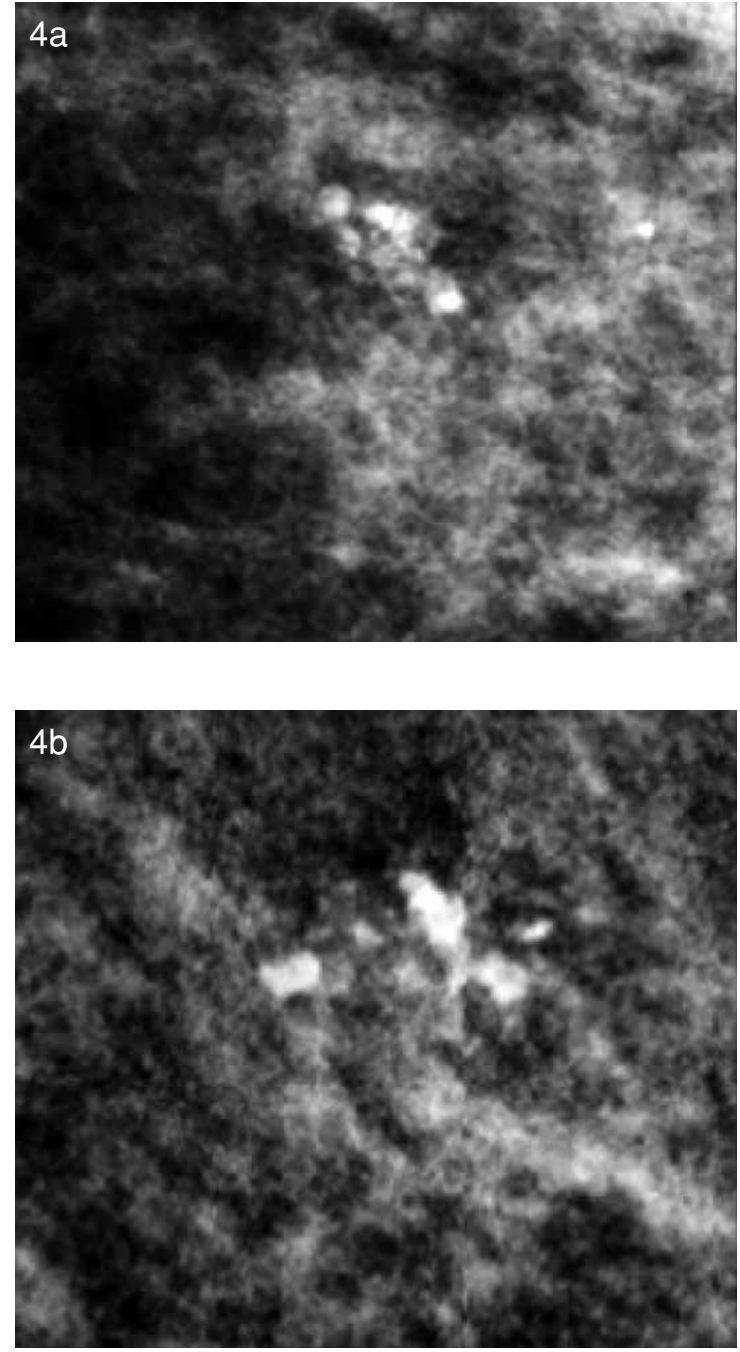

Figura $4 a$ y 4 b. Magnificaciones en proyecciones CC y LT de grupo de microcalcificaciones catalogadas como $B I-R A D S 4 A$, la histología de la BE resultó lesión de alto riesgo (HDA).

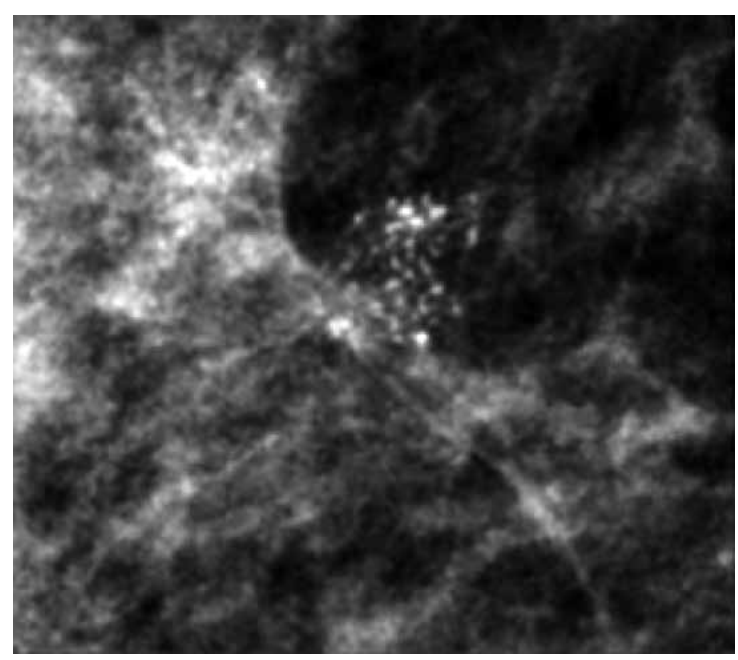

Figura 5. Magnificación en proyección $L T$ de grupo de microcalcificaciones BI-RADS 4A, la histología de la $B E$ fue maligna (carcinoma ductal in situ).
De las 16 lesiones malignas el 81,3\% correspondieron a CDIS y un $18,7 \%$ a carcinoma ductal infiltrante (CDI) (Gráfico 2), todas ellas se sometieron a cirugía, pero no contamos con la biopsia definitiva en una paciente que se operó en otro centro. La histología definitiva en las 15 pacientes restantes fue concordante en el $100 \%$ de los casos con el de la $\mathrm{BE}$, destacando incluso que en el $31,3 \%(5 / 16)$ no hubo tumor residual en la biopsia quirúrgica (Tabla II).



Gráfico 2.

Tabla II. Concordancia histológica en lesiones malignas asociadas a microcalcificaciones $4 \mathrm{~A}$

\begin{tabular}{|clcc|}
$\begin{array}{l}\text { No de } \\
\text { pacientes }\end{array}$ & $\begin{array}{l}\text { Histología } \\
\text { BE }\end{array}$ & $\begin{array}{l}\text { Histología } \\
\text { definitiva }\end{array}$ & Concordancia \\
\hline 1 & CDIS & sin dato & $\begin{array}{c}\text { atención } \\
\text { en otro centro } \\
\text { Sí }\end{array}$ \\
2 & CDIS & sin tu residual & CDI \\
3 & CDI & CDIS & Sí \\
4 & CDIS & sin tu residual & Sí \\
5 & CDIS & CDIS & Sí \\
6 & CDIS & sin tu residual & Sí \\
7 & CDI & sin tu residual & Sí \\
8 & CDIS & CDIS & Sí \\
9 & CDIS & CDIS & Sí \\
10 & CDIS & CDIS & Sí \\
11 & CDIS & CDIS & Sí \\
12 & CDIS & CDI & Sí \\
13 & CDI & CDIS & Sí \\
14 & CDIS & CDIS & Sí \\
15 & CDIS & sin tu residual & Sí \\
16 & CDIS & Total & 15 \\
& & &
\end{tabular}

Dentro de las 75 lesiones de alto riesgo, encontramos mayoritariamente HDA con el $52 \%$ (39) y AEP con el $30,7 \%$ (23), HDA asociada a AEP en el $12 \%$ (9). Otras lesiones de alto riesgo encontradas en menor porcentaje, fueron LIN II en 4\% (3) y la lesión mucocele like en 1,3\% (1) (Gráfico 3). 




Gráfico 3.

De las pacientes que tuvieron histología con lesiones de alto riesgo, contamos con información de la biopsia quirúrgica o seguimiento en 60 pacientes (80\%). En 50 casos fueron a cirugía de ampliación, en ellas en el $88 \%(44 / 50)$ la histología definitiva resultó concordante con la BE (Tabla III); en las 6 pacientes restantes, la biopsia quirúrgica demostró patología maligna; en dos casos CDI; en tres CDIS y en uno carcinoma lobulillar infiltrante, por lo que nuestra subestimación histológica en lesiones de alto riesgo alcanzó al $12 \%$.

En un grupo de 11 pacientes que tenían lesiones de alto riesgo en la $\mathrm{BE}$, se optó por seguimiento clínicoradiológico, en vez de cirugía. Hasta la fecha tienen un promedio de seguimiento de 24 meses (rango 6-47 meses), sin demostrarse nuevas alteraciones en los exámenes realizados. En las 14 pacientes restantes no contamos con información.

Tabla III. Concordancia histológica lesiones de alto riesgo.

\begin{tabular}{lc|} 
& Número \\
\hline Concordancia histológica & $44 / 50$ \\
Subestimación & 6 \\
Seguimiento clínico-radiológico & 11 \\
Pacientes sin histología disponible & 14 \\
Total lesiones alto riesgo & 75 \\
\end{tabular}

De las biopsias estereotáxicas que resultaron con histología benigna (70 casos), contamos con datos de seguimiento en el $80 \%$, es decir en 56 pacientes, con un promedio de seguimiento de 24,7 meses (4 a 66).

De las 70 lesiones benignas (Gráfico 4), la gran mayoría correspondió a cambios fibroquísticos asociados o no a hiperplasia epitelial y a cambios de células columnares en el $74 \%$.

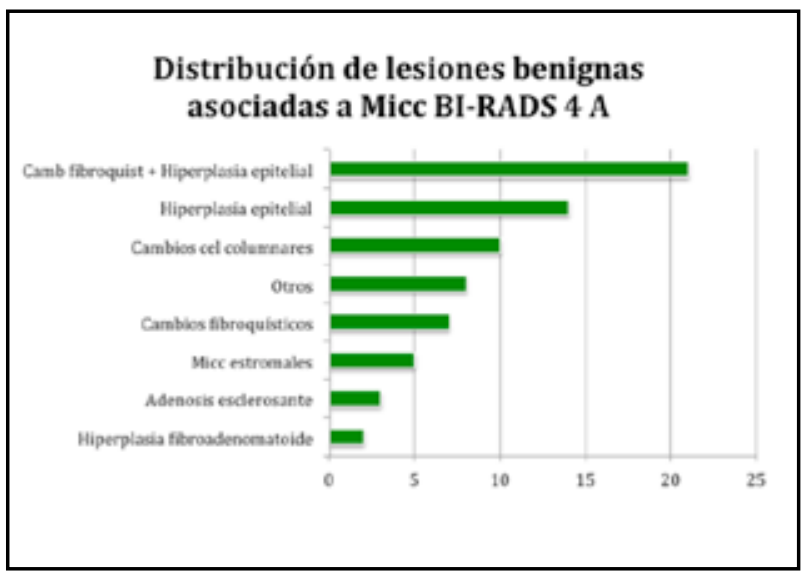

Gráfico 4.

\section{Discusión}

La subcategorización BI-RADS 4A ha permitido mejorar la pesquisa de lesiones sospechosas y mejorar el entendimiento entre clínicos y radiólogos. Sanders y cols ${ }^{(6)}$, Lazarus y cols ${ }^{(7)}$ y Bent y cols ${ }^{(8)}$, obtuvieron un VPP para las microcalcificaciones categoría BI-RADS $4 \mathrm{~A}$ de entre el $6 \%$ al $13 \%$, lo que es concordante con nuestra serie, en donde alcanzamos un VVP de $13 \%$.

El bajo porcentaje de microcalcificaciones categorizadas como BI-RADS 4A en nuestra casuística, que alcanza aproximadamente el $21,4 \%$, se explica a que empezamos con las BE en el año 1999 y la subcategorización del BI-RADS 4A aparece sólo en el año 2003. Actualmente consideramos indispensable el uso de la subclasificación 4A, B y C, en nuestras biopsias, tanto por fines de auditoría interna como de investigación.

Podemos destacar la excelente correlación de la BE con la biopsia definitiva, tanto en lesiones malignas como en lesiones de alto riesgo y la baja tasa de subestimación histológica la que alcanza sólo al $12 \%$ en cuanto a lesiones de alto riesgo versus cáncer. No hubo subestimación al evaluar cáncer in situ versus carcinoma ductal infiltrante, lo que atribuimos a la vasta experiencia de los radiólogos y al hecho de trabajar en un ambiente multidisciplinario, que nos permite casi siempre un adecuado seguimiento de las pacientes.

Concluimos, entonces, que las microcalcificaciones BI-RADS 4A, son de baja sospecha de malignidad, correspondiendo en su gran mayoría $(90 \%)$, a lesiones benignas.

La subdivisión en $4 \mathrm{~A}$ representa una herramienta que facilita un mejor manejo clínico de las pacientes, por lo que recomendamos su utilización. 


\section{Bibliografía}

1. Gajdos C, Tartter P, Bleiweiss I, et al. Mammographic appearance of nonpalpable breast cancer reflects pathologic characteristics. Ann Surg 2002; 235: 246 225.

2. De Roos MA, van der Vegt B, de Vries J, Wesselung J, de Bock GH. Pathological and biological differences between screen-detected and interval ductal carcinoma in-situ of the breast. Ann Surg Oncol 2007; 14: 20972104.

3. American College of Radiology. Breast Imaging Reporting and Data System, Breast Imaging Atlas, 4th edn. Reston, VA: American College of Radiology, 2003.

4. Lacquement MA, Mitchell D, Hollingsworth AB. Positive predictive value of the breast imaging reporting and data system. J Am Coll Surg 1999; 189: 34-40.

5. Orel SG, Kay N, Reynolds C, Sullivan DC. BI-RADS categorization as a predictor of malignancy. Radiology 1999; 211: 845-850.

6. Sanders MA, Roland L, Sahoo S. Clinical implications of subcategorizing BI-RADS 4 breast lesions associated with microcalcification: a radiology-pathology correlation study. Breast J. 2010; 16(1): 28-31. Epub 2009 Nov 19.

7. Lazarus E, Mainiero MB, Schepps B, et al. BI-RADS lexicon for US and mammography: interobserver variability and positive predictive value. Radiology 2006; 239: 385-391.

8. Bent CK, Bassett LW, D'Orsi CJ, Sayre JW. The positive predictive value of BI-RADS microcalcification descriptors and final assessment categories. AJR Am J Roentgenol. 2010; 194: 1378-1383. 